\title{
Effects of first-dose volume and exercise on the efficacy and tolerability of bowel preparations for colonoscopy in Chinese people
}

This article was published in the following Dove Press journal:

Therapeutics and Clinical Risk Management

I5 April 2016

Number of times this article has been viewed

Ying Qin

Wei Liu

Songbai Lin

Xiangfeng $\mathrm{Li}$

International Medical Services, Peking Union Medical College Hospital, Beijing, People's Republic of China
Correspondence: Songbai Lin International Medical Services, Peking Union Medical College Hospital, ShuaiFu Garden I, Dongcheng district, Beijing 100730, People's Republic of China

Tel +86 10 I86 I267 I8I5

Email lin_songbai@hotmail.com
Aim: This study was designed to compare the efficacy and tolerability of bowel preparations with and without the higher first-dose volume of polyethylene glycol (PEG) solution or exercise after drinking PEG solution in Chinese people.

Methods: A total of 330 participants who had a colonoscopy done in Peking Union Medical College Hospital were randomly and evenly assigned to three groups. Participants in Group A ingested $1 \mathrm{~L}$ PEG solution and then ingested $2 \mathrm{~L}$ PEG solution at a rate of $250 \mathrm{~mL}$ every 15 minutes. Participants in Group B ingested 3 L PEG solution at a rate of $250 \mathrm{~mL}$ every 15 minutes and then exercised more than 10 minutes after ingesting each liter of PEG solution. Participants in Group C ingested 3 L PEG solution at a rate of $250 \mathrm{~mL}$ every 15 minutes. Experienced gastrointestinal endoscopists rated the efficacy of bowel preparations based on the Boston Bowel Preparation Scale score. A questionnaire regarding participants' symptoms associated with bowel preparations was administered to evaluate participants' tolerability.

Results: The three groups had insignificant difference in the percentages of participants' symptoms including dizziness, nausea, stomach ache, bloating, and asthenia. However, the percentages of participants having hunger sensation, sleep disturbance, and anal discomfort were significantly higher in groups with the higher first-dose volume of PEG solution or exercise after drinking PEG solution than without them. The three groups had insignificant difference in the Boston Bowel Preparation Scale score.

Conclusion: Whether to add the higher first-dose volume of PEG solution and exercise after drinking PEG solution or not, all participants achieved a similar quality of bowel preparations. Bowel preparations without the additional first-dose volume of PEG solution or exercise after drinking PEG solution showed the advantage of high participant tolerability.

Keywords: bowel preparations for colonoscopy, efficacy, exercise, first-dose volume, polyethylene glycol, tolerability

\section{Introduction}

Colorectal cancer is one of the most harmful cancers on a global scale. ${ }^{1-3}$ Inspection by colonoscopy can bring about accurate detection and effective treatment of colorectal cancer. ${ }^{4,5}$ In addition, colonoscopy can also be used to detect other gastroenterological lesions including inflammatory bowel disease. ${ }^{6}$ Currently, polyethylene glycol (PEG) is recognized as the most important medicine used for bowel preparations for colonoscopy, and only it has been chosen by the latest Chinese guidelines on bowel preparations. ${ }^{7-10}$ Many bowel preparation methods using PEG exist, and the optimal method has the best efficacy and tolerability. ${ }^{11-13}$ Some studies have observed the effects of first-dose volume and exercise on the efficacy and tolerability of bowel preparations 
for colonoscopy, and the best option is still debatable. ${ }^{14}$ This study was designed to compare the efficacy and tolerability of bowel preparations with and without the higher first-dose volume of PEG solution or exercise after drinking PEG solution in a Chinese cohort in Beijing.

\section{Methods}

\section{Study participants}

After excluding participants with serious anorectal suppurative inflammation or painful lesions, acute enteritis, radioactive colitis, ischemic bowel disease, gastrointestinal stenosis, hemorrhage or surgery, pelvic surgery or pelvic inflammatory diseases, enterobrosis, peritonitis, pregnancy, menstruation, and cardiovascular, cerebrovascular, hepatic, renal, or mental diseases, 330 participants older than 18 years who were prepared to get a colonoscopy done in Peking Union Medical College Hospital between January and October 2014 were enrolled in this study. This study was approved by the Ethics Committee of Peking Union Medical College Hospital and conducted based on the Declaration of Helsinki. Written participant consent has been obtained in this study.

\section{Bowel preparations}

Participants meeting the study eligibility criteria were randomly divided into three groups (groups A-C), and each group consisted of 110 participants. Participants in Group A ingested $1 \mathrm{~L}$ PEG solution (one bag of PEG) at 8 pm the day before colonoscopy, and then ingested 2 L PEG solution (two bags of PEG) at a rate of $250 \mathrm{~mL}$ every 15 minutes. Participants in Group B ingested 3 L PEG solution (three bags of PEG) at a rate of $250 \mathrm{~mL}$ every 15 minutes at $8 \mathrm{pm}$ the day before colonoscopy and then exercised more than 10 minutes after ingesting each liter of PEG solution. Participants in Group C ingested 3 L PEG solution (three bags of PEG) at a rate of $250 \mathrm{~mL}$ every 15 minutes at $8 \mathrm{pm}$ the day before colonoscopy. All participants were banned from eating high-residue diets the day before colonoscopy and from discussing with endoscopists on the bowel preparation group to which they had been assigned.

\section{Efficacy and tolerability of bowel preparations}

Experienced gastrointestinal endoscopists rated the efficacy of bowel preparations based on the Boston Bowel Preparation Scale (BBPS) score, ${ }^{15}$ a 4-point scoring system applied to each of the three regions of the colon: right colon, transverse colon, and left colon. The points were assigned as follows: 0 (inadequate: unprepared colon segment with the mucosa not visible because of solid stool that could not be cleared); 1 (poor: portion of the mucosa of the colon segment visible, but other areas of the colon segment not well visualized because of staining, residual stool, or opaque liquid); 2 (good: minor amount of residual staining, small fragments of stool or opaque liquid, but well visualized mucosa of the colon segment); and 3 (excellent: entire mucosa of the colon segment well visualized with no residual staining, small fragments of stool, or opaque liquid). Each region of the colon received a segment score from 0 to 3 , and these segment scores were summed up for obtaining a total BBPS score ranging from 0 (completely unprepared) to 9 (perfect). Also, a questionnaire regarding participants' symptoms associated with bowel preparations was administered before colonoscopy to evaluate participants' tolerability. Participants were asked if they had any of the following symptoms: hunger sensation, sleep disturbance, dizziness, nausea, stomach ache, bloating, asthenia, and anal discomfort.

\section{Statistical analysis}

Statistical analysis was carried out with Statistical Package for the Social Sciences 17.0 (SPSS Inc., Chicago, IL, USA). Continuous variables were described as mean (standard deviation) or median (interquartile range) and categorical variables as count (percentage). Percentages of participants' symptoms as well as the BBPS scores were compared among the three groups with $\chi^{2}$ tests. Hypotheses were statistically significant with $P$-value $<0.05$.

\section{Results}

Age, male-to-female ratio, and stool situation in the last 3 months were comparable among the three groups $(P>0.05$ for all). The three groups had insignificant difference in the percentages of participants' symptoms including dizziness, nausea, stomach ache, bloating, and asthenia $(P>0.05$ for all). However, the percentages of participants having hunger sensation, sleep disturbance, and anal discomfort were significantly higher in groups with the higher first-dose volume of PEG solution or exercise after drinking PEG solution than without them $(P<0.05$ for all) (Table 1$)$. The three groups had insignificant difference in the BBPS score $(P>0.05)$ (Table 2).

\section{Discussion}

The BBPS score with good reproducibility was applied to assess the efficacy of bowel preparations in this analysis. ${ }^{16}$ All groups in our analysis showed similar efficacy of bowel preparations based on the BBPS score, suggesting that bowel 
Table I General characteristics of participants and participant tolerability of bowel preparations for colonoscopy in different groups

\begin{tabular}{|c|c|c|c|c|}
\hline Characteristics & Group $A^{a}(\%)$ & Group B ${ }^{\mathrm{b}}(\%)$ & Group Cc (\%) & $P$-value \\
\hline \multicolumn{5}{|l|}{ General features } \\
\hline Age, years (interquartile range) & $5 I(43-57)$ & 50 (45-59) & $51(44-62)$ & 0.644 \\
\hline Male & $66(60.0)$ & $71(64.5)$ & $66(60.0)$ & 0.726 \\
\hline Stool situation in the last 3 months & & & & 0.485 \\
\hline Regular bowel & $82(74.5)$ & 89 (80.9) & $92(83.6)$ & - \\
\hline Constipation & II (I0.0) & $9(8.2)$ & $9(8.2)$ & - \\
\hline Diarrhea & $17(15.5)$ & $12(10.9)$ & $9(8.2)$ & - \\
\hline \multicolumn{5}{|l|}{ Tolerability } \\
\hline Hunger sensation & $38(34.5)$ & $24(21.8)$ & $23(20.9)$ & 0.035 \\
\hline Sleep disturbance & $45(40.9)$ & $66(60.0)$ & $22(20.0)$ & $<0.001$ \\
\hline Dizziness & $13(\mid 1.8)$ & $17(15.5)$ & $10(9.1)$ & 0.349 \\
\hline Nausea & $55(50)$ & $54(49.1)$ & $42(38.2)$ & 0.147 \\
\hline Stomach ache & $7(6.4)$ & $6(5.5)$ & $6(5.5)$ & 0.946 \\
\hline Bloating & $51(46.4)$ & $50(45.5)$ & $44(40.0)$ & 0.589 \\
\hline Asthenia & $32(29.1)$ & $25(22.7)$ & $21(19.1)$ & 0.210 \\
\hline Anal discomfort & $43(39.1)$ & $38(34.5)$ & $24(2 \mid .8)$ & 0.017 \\
\hline
\end{tabular}

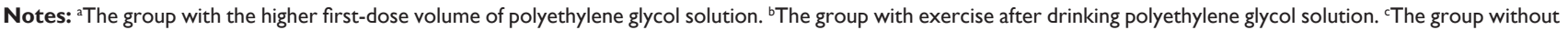
the higher first-dose volume of polyethylene glycol solution or exercise after drinking polyethylene glycol solution.

preparations without the higher first-dose volume of PEG solution or exercise after drinking PEG solution are not inferior to those with these methods. An earlier study considered nonexercise as a risk factor for poor bowel cleansing and that exercises such as walking might improve bowel cleansing. ${ }^{14}$ But this study showed that exercise was a method leading to significant discomfort without better bowel cleansing in Chinese people. In other words, exercise had no significant influence on the efficacy of bowel preparations. Meanwhile, we realized that bowel preparations without the higher firstdose volume of PEG solution provided a distinct advantage over other methods when efficacy and tolerability were considered collectively.

Although the symptoms related to bowel preparations, including dizziness, nausea, stomach ache, bloating, and asthenia, were similar among the three groups, other symptoms including hunger sensation, sleep disturbance, and anal discomfort were more common due to the influence of the higher first-dose volume of PEG or exercise after drinking PEG solution in this study. The process of bowel preparations itself was uncomfortable due to drinking a large amount of PEG solution and subsequent severe diarrhea until the intestine was clean. It is not hard to understand that the higher first-dose volume of PEG solution or exercise after drinking PEG solution results in associated discomfort and symptoms. The larger amount of colon-cleansing preparation solutions often causes dissatisfaction among participants, and the larger first-dose volume of PEG solution has similar problem. ${ }^{17,18}$ The higher first-dose volume of PEG solution and exercise after drinking PEG were unable to improve the efficacy of bowel preparations and were not as well tolerated and safe as the methods without them in this study, and thus, we do not suggest the additional firstdose volume of PEG solution and exercise after drinking

Table 2 Efficacy of bowel preparations for colonoscopy according to the Boston Bowel Preparation Scale score in different groups

\begin{tabular}{|c|c|c|c|c|}
\hline Boston Bowel Preparation Scale score & Group $A^{a}(\%)$ & Group B ${ }^{\mathrm{b}}(\%)$ & Group $\mathbf{C}^{c}(\%)$ & $P$-value \\
\hline 0 & $\mathrm{I}(0.9)$ & $0(0)$ & $0(0)$ & 0.495 \\
\hline I & $\mathrm{I}(0.9)$ & $2(1.8)$ & $0(0)$ & - \\
\hline 2 & $4(3.6)$ & $4(3.6)$ & $\mathrm{I}(0.9)$ & - \\
\hline 3 & $8(7.3)$ & $16(14.5)$ & $8(7.3)$ & - \\
\hline 4 & $3(2.7)$ & $6(5.5)$ & $12(10.9)$ & - \\
\hline 5 & $16(14.5)$ & $16(14.5)$ & $16(14.5)$ & - \\
\hline 6 & $25(22.7)$ & $20(18.2)$ & $21(19.1)$ & - \\
\hline 7 & $12(10.9)$ & $10(9.1)$ & I3 (I I.8) & - \\
\hline 8 & $9(8.2)$ & $8(7.3)$ & $10(9.1)$ & - \\
\hline 9 & 31 (28.2) & $28(25.5)$ & $29(26.4)$ & - \\
\hline
\end{tabular}

Notes: ${ }^{2}$ The group with the higher first-dose volume of polyethylene glycol solution. ${ }^{\mathrm{b}}$ The group with exercise after drinking polyethylene glycol solution. ${ }^{\mathrm{c}}$ The group without the higher first-dose volume of polyethylene glycol solution or exercise after drinking polyethylene glycol solution. 
PEG solution as the standard methods in clinical practice for Chinese people.

This study had one weakness. Participants' characteristics including weight were not considered in this study. However, these characteristics of the participants, especially weight, are not significant factors that directly affect the conduct of bowel preparations for colonoscopy and have no obvious influence on the results of this study.

\section{Conclusion}

This study demonstrated that whether the higher first-dose volume of PEG solution or exercise was added after drinking PEG solution or not, all participants achieved a similar quality of bowel preparations. Bowel preparations without the additional first-dose volume of PEG solution or exercise after drinking PEG solution showed the advantage of high participant tolerability.

\section{Disclosure}

The authors report no conflicts of interest in this work.

\section{References}

1. Bray F, Ren JS, Masuyer E, Ferlay J. Global estimates of cancer prevalence for 27 sites in the adult population in 2008. Int J Cancer. 2013;132: $1133-1145$.

2. van Leersum NJ, Janssen-Heijnen ML, Wouters MW, et al. Increasing prevalence of comorbidity in patients with colorectal cancer in the South of the Netherlands 1995-2010. Int J Cancer. 2013;132:2157-2163.

3. Maringe C, Mangtani P, Rachet B, Leon DA, Coleman MP, dos Santos Silva I. Cancer incidence in South Asian migrants to England, 1986-2004: unraveling ethnic from socioeconomic differentials. Int J Cancer. 2013; 132:1886-1894.

4. Brenner H, Chang-Claude J, Jansen L, Seiler CM, Hoffmeister M. Colorectal cancers occurring after colonoscopy with polyp detection: sites of polyps and sites of cancers. Int J Cancer. 2013;133:1672-1679.
5. Lee SH, Park YK, Lee DJ, Kim KM. Colonoscopy procedural skills and training for new beginners. World J Gastroenterol. 2014;20: 16984-16995.

6. Lei P, Gu F, Hong L, et al. Pediatric colonoscopy in South china: a 12-year experience in a tertiary center. PLoS One. 2014;9:e95933.

7. Belsey J, Crosta C, Epstein O, et al. Meta-analysis: The relative efficacy of oral bowel preparations for colonoscopy 1985-2010. Aliment Pharmacol Ther. 2012;35:222-237.

8. Hassan C, Bretthauer M, Kaminski MF, et al. Bowel preparation for colonoscopy: European Society of Gastrointestinal Endoscopy (ESGE) guideline. Endoscopy. 2013;45:142-150.

9. Chinese Society for Gastrointestinal Endoscopy. Chinese consensus on bowel preparation before endoscopy. Chinese J Pract Intern Med. 2013;33:705-707.

10. Lee SH, Lee DJ, Kim KM, et al. Comparison of the efficacy and safety of sodium phosphate tablets and polyethylene glycol solution for bowel cleansing in healthy Korean adults. Yonsei Med J. 2014;55: $1542-1555$.

11. Martens P, Bisschops R. Bowel preparation for colonoscopy: efficacy, tolerability and safety. Acta Gastroenterol Belg. 2014;77:249-255.

12. Hillyer GC, Basch CH, Lebwohl B, et al. Shortened surveillance intervals following suboptimal bowel preparation for colonoscopy: results of a national survey. Int J Colorectal Dis. 2013;28:73-81.

13. Lebwohl B, Kastrinos F, Glick M, Rosenbaum AJ, Wang T, Neugut AI. The impact of suboptimal bowel preparation on adenoma miss rates and the factors associated with early repeat colonoscopy. Gastrointest Endosc. 2011;73:1207-1214.

14. Kim HS, Park DH, Kim JW, et al. Effectiveness of walking exercise as a bowel preparation for colonoscopy: a randomized controlled trial. Am J Gastroenterol. 2005;100:1964-1969.

15. Lai EJ, Calderwood AH, Doros G, Fix OK, Jacobson BC. The Boston bowel preparation scale: a valid and reliable instrument for colonoscopyoriented research. Gastrointest Endosc. 2009;69:620-625.

16. Calderwood AH, Schroy PC 3rd, Lieberman DA, Logan JR, Zurfluh M, Jacobson BC. Boston Bowel Preparation Scale scores provide a standardized definition of adequate for describing bowel cleanliness. Gastrointest Endosc. 2014;80:269-276.

17. Golub R, Kerner B, Wise Jr W, et al. Colonoscopic bowel preparations which one? Dis Colon Rectum. 1995;38:594-599.

18. Kastenberg D, Chasen R, Choudhary C, et al. Efficacy and safety of sodium phosphate tablets compared with PEG solution in colon cleansing: two identically designed, randomized, controlled, parallel group, multicenter phase III trials. Gastrointest Endosc. 2001;54:705-713.
Therapeutics and Clinical Risk Management

\section{Publish your work in this journal}

Therapeutics and Clinical Risk Management is an international, peerreviewed journal of clinical therapeutics and risk management, focusing on concise rapid reporting of clinical studies in all therapeutic areas, outcomes, safety, and programs for the effective, safe, and sustained use of medicines. This journal is indexed on PubMed Central, CAS,

\section{Dovepress}

EMBase, Scopus and the Elsevier Bibliographic databases. The manuscript management system is completely online and includes a very quick and fair peer-review system, which is all easy to use. Visit http://www.dovepress.com/testimonials.php to read real quotes from published authors. 